\title{
A new readout system for bolometers with improved low frequency stability
}

\author{
S. Gaertner ${ }^{1}$, A. Benoît ${ }^{2}$ ，J.-M. Lamarre ${ }^{3}$, M. Giard ${ }^{1}$ ， J.-L. Bret ${ }^{2}$ ，J.-P. Chabaud ${ }^{1}$, F.-X. Désert ${ }^{3}$, \\ J.-P. Faure ${ }^{2}$ ， G. Jegoudez ${ }^{3}$ ， J. Landé ${ }^{1}$ ， J. Leblanc ${ }^{3}$ ， J.-P. Lepeltier ${ }^{3}$ ， J. Narbonne ${ }^{1}$ ， M. Piat ${ }^{3}$, \\ R. Pons ${ }^{1}$, G. Serra ${ }^{1}$, and G. Simiand ${ }^{2}$ \\ 1 Centre d'Etude Spatiale des Rayonnements, 9 avenue du Colonel Roche, BP. 4346, F-31028 Toulouse Cedex 04, France \\ 2 Centre de Recherche des Très Basses Températures, 25 avenue des Martyrs, BP. 166, F-38042 Grenoble Cedex 09, France \\ ${ }^{3}$ Institut d'Astrophysique Spatiale, Université Paris XI, Bât. 121, F-91405 Orsay Cedex, France
}

Received December 23, 1996; accepted February 24, 1997

\begin{abstract}
A new readout electronic system for bolometers is presented in this paper. The bolometer resistance is measured in a bridge with a capacitive load, using a periodic square wave bias current. The bias voltages at both ends of the bridge are balanced in order to keep the middle point around zero. Only changes around this zero value are amplified and detected synchronously with the bias signal. These features shift the measurement frequency out of the electrical low frequency noises (JFETs), and reduces the dynamics required from the amplification chain. The bias voltages are fully controlled by computer, and the lockin detection is digital. This readout electronic has many advantages over previous ones. In particular, it proved to be able to read the total power of the radiation reaching the bolometer, and to perform measurements down to low frequencies $(\leq 0.1 \mathrm{~Hz})$ without significant additional noise. These features open new observation strategies such as full sky scanning to bolometer instruments on board future submillimetre space projects having high thermal background levels (warm telescope). The different steps of the development of this new readout electronics on the ground-based Diabolo experiment are described, the performances reached are discussed, and a version suitable for the readout of the arrays of bolometers on the COBRAS/SAMBA satellite mission is presented.
\end{abstract}

Key words: instrumentation: detectors; instrumentation: photometer — methods: data analysis methods: numerical

\section{Introduction}

Bolometers are used in a very wide range of frequencies, from X-rays to millimetre waves. This results

Send offprint requests to: S. Gaertner, gaertner@cesr.cnes.fr from the principles of thermal detection: the temperature changes of a radiation absorber are measured by a thermometer. The efficiency of this process depends on that of the radiation absorption. The theory of bolometers has been developed and refined during the last half-century (Jones 1953; Low 1961; Mather 1982, 1984). The most currently accepted assumptions are that the temperature changes are measured using a thermistor attached to the radiation absorber and electrically connected to a load resistor (Fig. 1, left).

This scheme has been used successfully in a large number of applications, especially in instruments dedicated to astronomical measurements in the far infrared and the submillimetre wavelength ranges. Nevertheless, low frequency noises in electronic components used to amplify the signal, generally gathered under the denomination of $1 / \mathrm{f}$ noise, make it impossible to use this principle for the measurement of DC optical signals. Sky chopping, which consists in alternately orientating the beam towards two neighbour directions, and measuring the signal difference with a lock-in amplifier, shifts the measuring frequency around that of the sky chopping, out of the $1 / \mathrm{f}$ noise frequency range. This has also the advantage of removing foreground emissions common to both positions of the beam, such as those of the atmosphere and the telescope. The disadvantages are that the measurement is now only a differential one (measuring the brightness difference between two fields of view), and that the implementation of a beam switching is sometimes a major technical difficulty, needing for example additional mirrors, and a poorer optical efficiency. Other electrical schemes have been studied to measure the impedance of the thermistor with an AC bridge circuit, which shifts the measuring domain outside the $1 / \mathrm{f}$ noise domain (Rieke et al. 1989; Devlin et al. 1993). In these systems, the bolometer to be measured is mounted in a bridge with another bolometer. In order to perform a measurement of the total power, the 

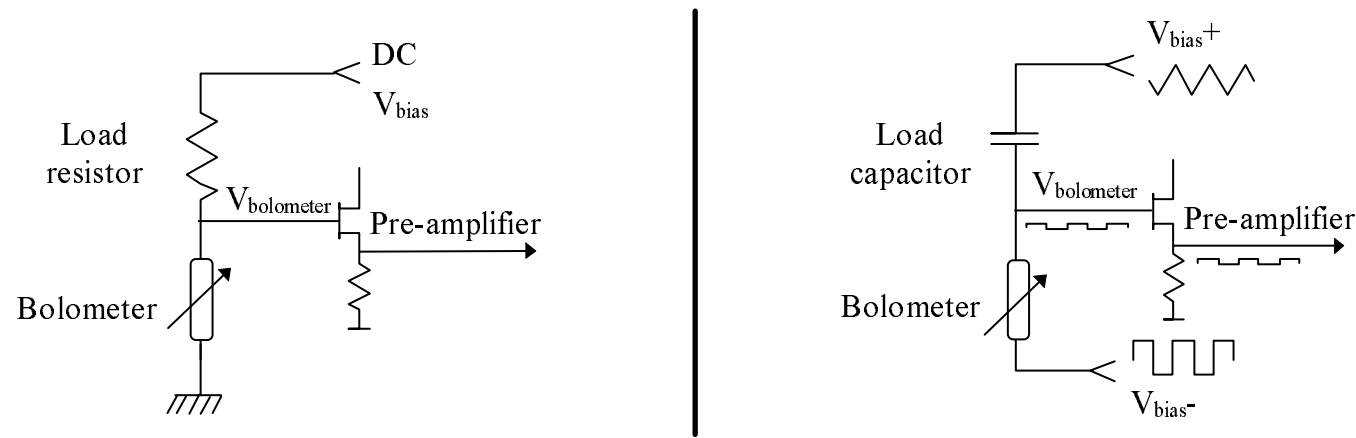

Fig. 1. Schemes showing the principles of a conventional DC bias (left), and of a balanced periodic square wave bias with a capacitive load (right)

comparison bolometer has to be blind. Both bolometers need to have similar impedances. This can be achieved if the background photon flux on the bolometer to be measured is negligible. This was the case for the IRTS instrument (Murakami et al. 1996), the cryogenically cooled Japanese infrared telescope on which the system of Delvin et al. (1993) has been implemented. However in the case of the COBRAS/SAMBA project (Bersanelli et al. 1996), an important thermal background photon flux from the telescope $(T \sim 100 \mathrm{~K})$ reaches the bolometer. The blind and open bolometer will have very different impedances which makes impossible the balance of the bridge. To overcome this problem one usually includes in the bridge two bolometers of the focal plane corresponding to two different sky positions (Wilbanks et al. 1990). Such a measurement is differential and not appropriate to a survey mission like COBRAS/SAMBA which has to measure all the angular frequencies of the sky emission. Another solution would be to artificially heat the blind bolometer (Lange, private communication). The problem then is to provide a very stable heating device.

The readout system (see Fig. 1, right) presented in this paper allows total power single pixel measurements at the focus of a warm telescope, accross a wide electrical frequency band. It is new in several respects: 1) The dynamics needed is kept reasonable thanks to a zero measurement method obtained with balanced bias voltages. 2) The load impedance is a capacitor (instead of a resistor), and then free of Johnson noise. 3) The whole system is digitally controlled, and the bias parameters can be tuned by a computer. This system has been developed by steps and implemented on the Diabolo experiment (Benoît et al. 1996 and Désert et al. 1996) with bolometers (Coron 1976) cooled at $0.1 \mathrm{~K}$. This instrument has been successfully used for high sensitivity ground-based astronomical observations in the millimetre domain. In the next section of this paper (Sect. 2), the principle of the balanced bias with square waves is detailed. In Sect. 3, the advantages of a capacitive load are shown, and a feed- back system used to control the bias level is discussed. Section 4 is dedicated to the description of a computer control of this bias system, while Sect. 5 analyses and discusses its performances. The architecture of a space qualifiable version, suitable for the High Frequency Instrument of COBRAS/SAMBA is described in Sect. 6. In the conclusion (Sect. 7), the advantages of this new readout system are discussed.

\section{A balanced bias with two square waves and a resistive load}

The choice of a periodic bias results from the desire to avoid the contamination of the useful signal by low frequency noises of electrical origin, which is critical for instruments that cannot benefit from a high frequency optical modulation, such as the COBRAS/SAMBA project (Bersanelli et al. 1996). Unlike the systems proposed by Lange et al. (1996), which use a sine wave bias, the system presented here uses a square wave bias. This means that the electrical power dissipated in the bolometer is constant, and therefore the temperature of the bolometer does not oscillate. In consequence, there is no low frequency limit associated with the thermal time constant of the bolometer, and the well developed theory of DC biased bolometers can be directly used. A second original feature of this new system is that the bias is applied with opposite phases to the bolometer and to the load resistor. It is then possible to tune the amplitude of both bias voltages to obtain a signal near zero at the middle point and an optimal operating bias of the bolometer.

In practice, two very stable square wave voltages of opposite sign (see Fig. 2, left) are applied to both ends of the bolometer-load resistor bridge, and the middle point is connected to the pre-amplifier. The value of the load resistor $R_{\mathrm{L}}$ has to be very large in comparison with the bolometers resistance $R_{\mathrm{B}}\left(R_{\mathrm{L}} \gg R_{\mathrm{B}}\right)$, in order to determine a nearly constant electric current (Mather 1982). It is possible to adjust the two bias voltages $V_{\mathrm{L}}$ and $V_{\mathrm{B}}$, in such a way that the bridge is well-balanced and that the 


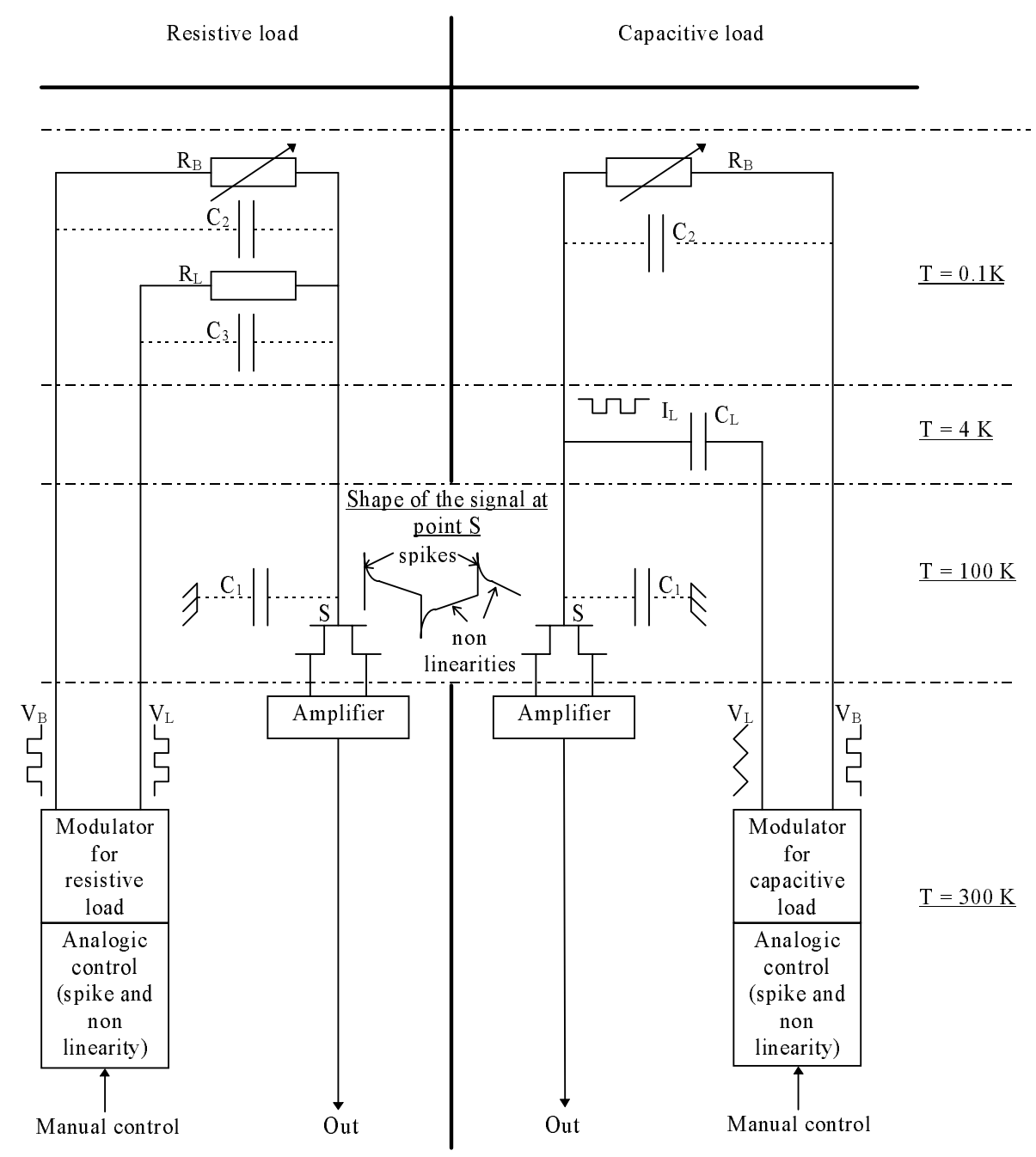

Fig. 2. Electric modulation system for bolometers with a resistive load (Diabolo, May 1994; left) and with a capacitance load (Diabolo, March 1995; right)

bolometer is optimally biased for the current application. The voltage $U_{\mathrm{S}}$ at point $\mathrm{S}$ is then equal to zero. When the bridge is not well-balanced, due for example to a change in the incoming radiation, the voltage at point $\mathrm{S}$ is given by:

$U_{\mathrm{S}}=\frac{V_{\mathrm{L}} R_{\mathrm{B}}-V_{\mathrm{B}} R_{\mathrm{L}}}{R_{\mathrm{B}}+R_{\mathrm{L}}}$

It is then possible to measure the small changes of the voltage at point $\mathrm{S}$ due to the small fluctuations of the bolometers resistor i.e., at first order:

$U_{\mathrm{S}}=\frac{R_{\mathrm{L}}\left(V_{\mathrm{L}}+V_{\mathrm{B}}\right)}{\left(R_{\mathrm{B}}+R_{\mathrm{L}}\right)^{2}} \delta R_{\mathrm{B}}$

Since $V_{\mathrm{L}}$ and $V_{\mathrm{B}}$ are square waves, $U_{\mathrm{S}}$ is also a square wave that can be amplified by a conventional pre-amplifier which includes as a first stage a JFET at low temperature $(T<100 \mathrm{~K})$ used as a follower. However, parasitic capacitances $\left(C_{1}, C_{2}\right.$ and $C_{3}$, due to the resistors and the wires; see Fig. 2, left) introduce transients (spikes) in $U_{\mathrm{S}}$ at each step of the square wave, even when the bridge is balanced. For a half-period of the bias, the shape of these spikes is given by the following equation:

$$
\begin{aligned}
U_{\mathrm{S}}(t) & =\frac{V_{\mathrm{L}} R_{\mathrm{B}}}{S_{\mathrm{R}}}\left[1+\left(\frac{C_{2} S_{\mathrm{R}}}{R_{\mathrm{B}} S_{\mathrm{C}}}-1\right) \exp \left(-\frac{S_{\mathrm{R}}}{P_{\mathrm{R}} S_{\mathrm{C}}} t\right)\right] \\
& -\frac{V_{\mathrm{B}} R_{\mathrm{L}}}{S_{\mathrm{R}}}\left[1+\left(\frac{C_{3} S_{\mathrm{R}}}{R_{\mathrm{L}} S_{\mathrm{C}}}-1\right) \exp \left(-\frac{S_{\mathrm{R}}}{P_{\mathrm{R}} S_{\mathrm{C}}} t\right)\right] .
\end{aligned}
$$

where $U_{\mathrm{S}}(t)$ is the output voltage at point $\mathrm{S}$ and, $S_{\mathrm{R}}, P_{\mathrm{R}}$ and $S_{\mathrm{C}}$ are equal to:

$S_{\mathrm{R}}=R_{\mathrm{B}}+R_{\mathrm{L}}, P_{\mathrm{R}}=R_{\mathrm{B}} \times R_{\mathrm{L}}$ and $S_{\mathrm{C}}=C_{1}+C_{2}+C_{3}$.

The resulting spike is minimum when the following condition is fulfilled: $C_{2} R_{\mathrm{L}}=C_{3} R_{\mathrm{B}}$. This condition is 
generally not verified, but can be approached by addition of a capacitance. In practice, it has been found simpler to control the shape of the bias voltage in order to minimize the spikes and the non linearities in the output signal (see the shape of the signal at point $\mathrm{S}$ in Fig. 2). If a residual transient still exists, it can be discarded from the handled data by introducing a blank delay before integration of the output signal at each half period in the lock-in amplifier.

\section{Introducing capacitive loads and a feedback loop}

As will be shown thereafter, replacing the load resistor by a capacitance (see Fig. 2, right) improves the system. In this case, the capacitance is polarized by a triangular wave voltage, which produces a square wave current. This triangular voltage is built from the square voltage by an active integrator using an operational amplifier. The advantages of this substitution are significant. First of all, the capacitance does not produce any significant heat and is not a source of Johnson noise. It is not any more necessary to put this element on the coldest stage of the cryostat, for example at $T=0.1 \mathrm{~K}$. So, only two wires per bolometer reach the coldest stage (instead of three wires for a load resistor) which may prove a significant advantage at very low temperatures. The capacitance has a large input impedance: for $10 \mathrm{pF}$, the value of the impedance at $100 \mathrm{~Hz}$ is equal to $200 \mathrm{M} \Omega$, which is, of course, higher than the bolometer resi stors ( 1 to $40 \mathrm{M} \Omega$ ). Finally, it decreases the spike in the signal, becau se the parasitic capacitance $C_{3}$ is not active any more. The spike due to the bolometer remains. For this new system and for a half-period of bias, the shape of the signal at point $\mathrm{S}$ when the bridge is balanced reads:

$U_{\mathrm{S}}(t)=V_{\mathrm{B}} \frac{C_{2}}{S_{\mathrm{C}^{\prime}}} \exp \left(-\frac{1}{R_{\mathrm{B}} S_{\mathrm{C}^{\prime}}} t\right)$

where $S_{\mathrm{C}^{\prime}}=C_{\mathrm{L}}+C_{1}+C_{2}$. The bolometer resistance is, in this system and for a balanced bridge, determined by the ratio $V_{\mathrm{L}} / V_{\mathrm{B}}$. With a capacitive load, the shape of the bias signals can also be adjusted in order to minimize the effects of spikes and non linearities by adding a small triangular wave shape to the square wave voltage. In practice, it is necessary to adjust the current and the voltage on the bolometer in order to reach the best sensitivity and the equilibrium of the bridge. With analog electronics, the ratio between $V_{\mathrm{L}}$ and $V_{\mathrm{B}}$ can only be tuned by manually adjusting the voltages. To make the system workable for intensive astronomical observations, a feedback loop was implemented. The amplitude of the output signal, $U_{\mathrm{S}}$, is pre-amplified, lock-in amplified, integrated, and is used as the reference voltage to control the amplitudes of the square waves $V_{\mathrm{L}}$ and $V_{\mathrm{B}}$. The system works at constant $V_{\mathrm{L}} / V_{\mathrm{B}}$ so that at equilibrium, $U_{\mathrm{S}}=0$, a fixed value of the bolometer resistance is reached (see Fig. 3). Other principles could have been used, giving different laws for the bolometer impedance control. That one proved to be relevant for operational observations. It allows a direct power sensitivity calibration of the bolometer: if the radiation power changes, the electrical power due to the bolometer bias changes in opposite sense to compensate and keeps the bridge balanced. So, the measure of the bias amplitude gives a total power measurement. However, for the measured signal, the feedback control acts as a high pass filter which eliminates the very low frequencies, $f \ll f_{\text {loop}}$.

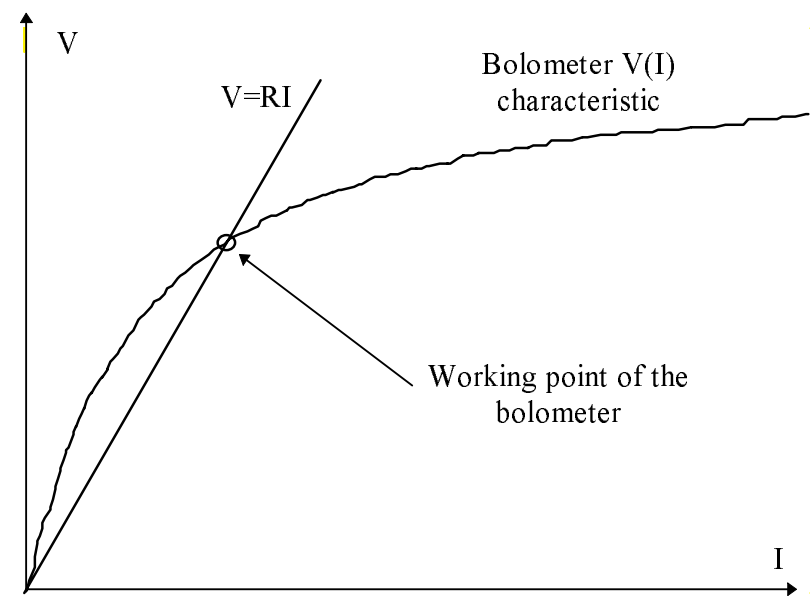

Fig. 3. Working point for a bolometer

Such a system has been used on the Diabolo photometer for the observations at the 2.7 meter MITO telescope in Italy (see Benoît et al. 1996). During the first campaign in May 1994, it worked with a resistive load and in March 1995, with a capacitive load. The system demonstrated its efficiency to avoid $1 /$ f noise. However, the operations and the data reduction after the observations allowed to identify two problems: 1) the need of manual adjustment of the impedance (ratio voltage/current), 2) the absence of direct record in the data of the total power, since manual tunings were not recorded. To solve this problem, the analog feed back loop has been replaced by a digital control of current and voltage, based on a real time digital analysis of the output signal, which is described in the next section.

\section{A computer controlled readout circuit}

In the new system, all parameters of the bias waves, including the compensation of spikes and non-linearities, are controlled by a computer. The "digital modulator" (see Fig. 4) producing the bias waves includes four high quality 12 bits Digital to Analog Converters (DACs), used to control the following parameters:

- DAC1: the amplitude of the bias voltage,

- DAC2: the intensity of the bias current, 


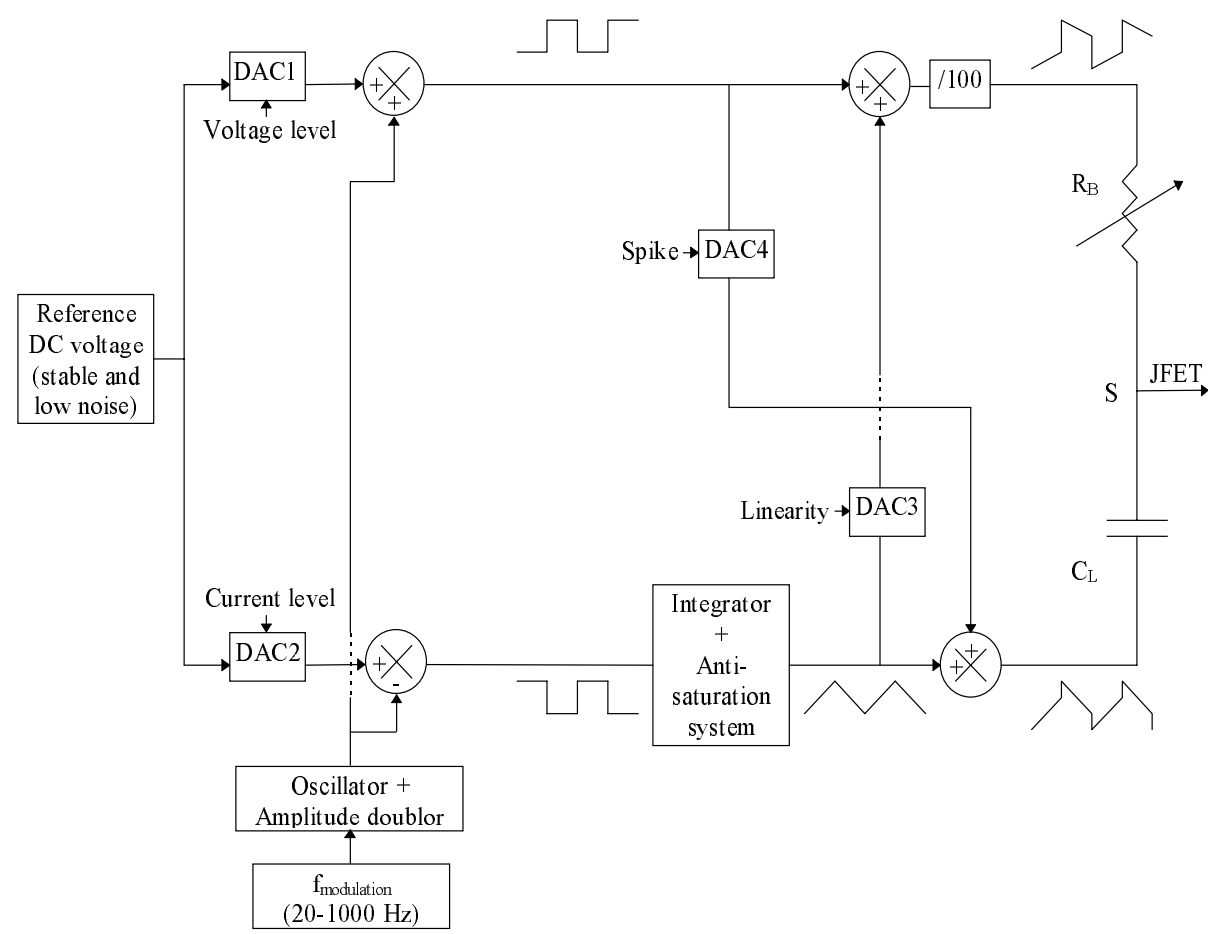

Fig. 4. Synoptic of the digital modulator

- DAC3: the slope of the bias voltage in each half period (this compensate for the triangular wave non linearities), - DAC4: the amplitude of a spike signal added to the bias current at the beginning of each half period (compensation of transients).

A point of prominent importance in this system is to use a reference voltage $\left(V_{\text {ref }}=10 \mathrm{~V}\right)$ with high stability and low noise, in order to feed the four DACs. The DC output level of each DAC crosses an inverting gate driven by the main clock, in order to obtain a square signal of very stable amplitude. The square bias voltage is divided by a resistance bridge (divided by 100) before being applied on the bolometer. Because a capacitance is used to drive the bolometer current, the analogic voltage signal is a triangular wave obtained after integration of the square wave. An anti-saturation system is added to the integrator to stabilize the average DC output level. This triangular voltage is applied to the load capacitor to generate the square bias current.

DAC3 and DAC4 allow to introduce some curvature and spike to the bias voltages in order to get a bolometer signal as flat as possible. DAC3 controls the addition of a fraction of the triangle signal to the square signal which will balance non linearities. DAC4 controls the addition of a fraction of the square current to the triangle signal. This is derived in anti-spikes current by the $C_{\mathrm{L}}$ capacitance.
Consequently, the bridge is well-balanced when:

$\frac{C_{\mathrm{L}} R_{\mathrm{B}}}{C_{\mathrm{i}} R_{\mathrm{i}}}=\frac{1}{100} \frac{V_{\mathrm{l}}}{I_{\mathrm{l}}}$

where $C_{\mathrm{i}}$ and $R_{\mathrm{i}}$ are the capacitance and the resistor of the integrator, $V_{1}$ and $I_{1}$ are the voltage and current levels on the bolometer. If the voltage and current levels are the same, and for $R_{\mathrm{B}}=40 \mathrm{M} \Omega, R_{\mathrm{i}}=200 \mathrm{k} \Omega$ and $C_{\mathrm{i}}=22 \mu \mathrm{F}$, the value of the load capacitance must be about $10 \mathrm{pF}$.

This system has been included in the electronics of the Diabolo experiment during an observation campaign on the IRAM 30 meter radio telescope at Pico Veleta (Spain) in December 1995. The Diabolo electronic system (see Fig. 5) is made of:

- a Box for Each Bolometer Output (BEBO), composed of:

- a "digital modulator" with a capacitive load,

- four DACs (12 bits),

- a pre-amplifier $(\times 100)$,

- a variable gain amplifier (from $\times 1$ to $\times 8000$ ),

- a 12 bits Analog to Digital Converter (ADC),

- a digital command logic,

- several opto-couplers isolating the analog part and the digital one, 


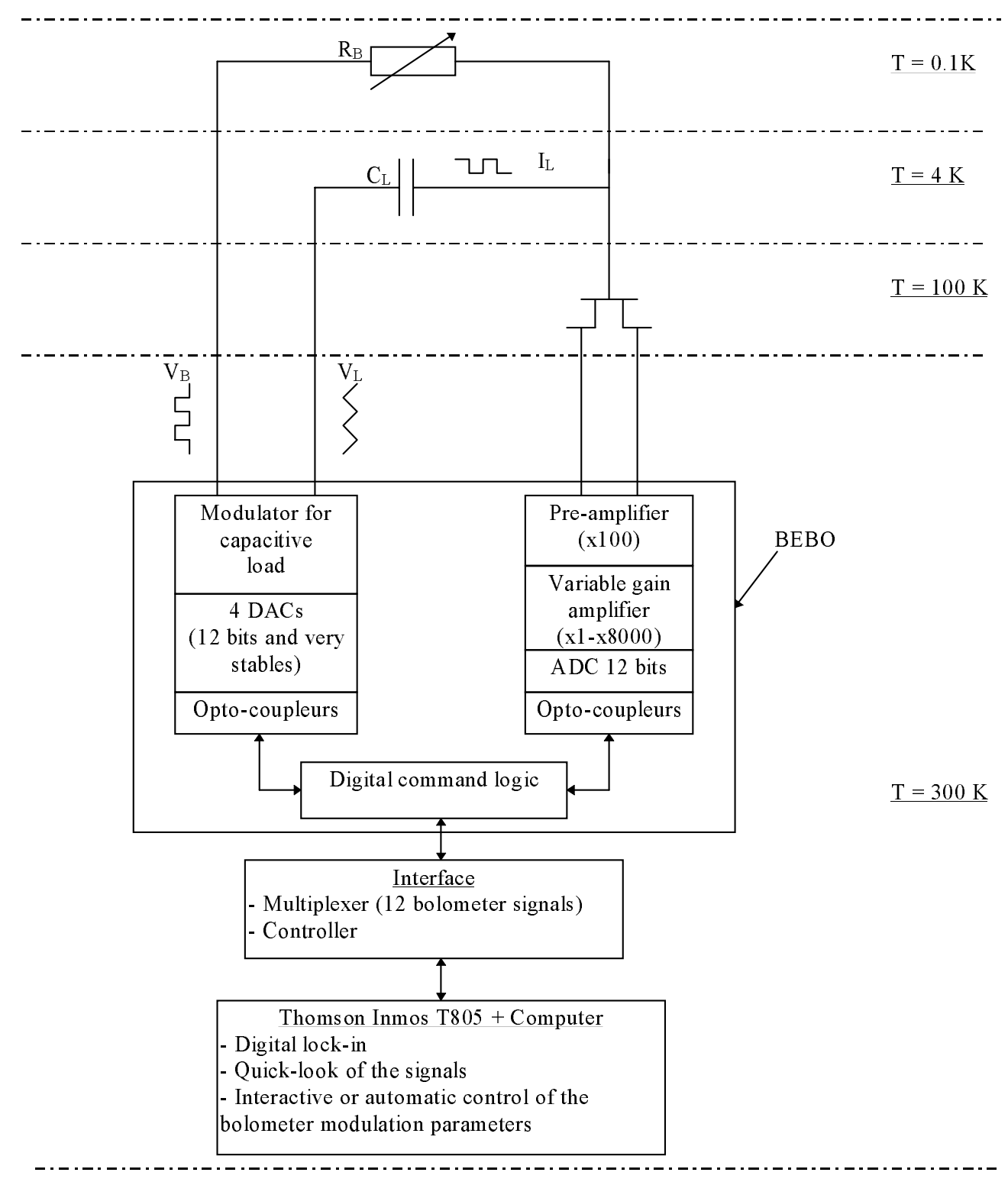

Fig. 5. Synoptic of the electronic system of the Diabolo experiment (December 1995)

- an interface, composed of:

- a multiplexer receiving 12 bolometer signals and driving the transmission receiver,

- a controller verifying the different signals and building the telemetry flow,

- a computer based on a Reduced Instruction Set Computer (RISC) processor, i.e. the Inmos Thomson T805, achieving the digital lock-in of the 12 bolometer signals, and a computer performing a quick-look of the signals and allowing interactive or automatic control of the bolometers bias parameters (DACs 1 through 4).

\section{Performances}

The electronic system of Diabolo has been tested with special configurations that allow to estimate the noise of the amplifier (Fig. 6), to control the stability of the bias amplitudes (Fig. 7), to measure the noise of the digital system with a bolometer simulator (Fig. 8), and to measure it with one bolometer of the Diabolo experiment (Fig. 9).

To evaluate the direct noise of the BEBO, the input is connected to the ground. The noise spectrum, after lockin, is measured with a maximum gain $\left(810^{5}\right)$ (see Fig. 6). The average noise level is about $0.5 \mathrm{nV} / \sqrt{\mathrm{Hz}}$, in good 
agreement with the expectation (JFET noise $0.3 \mathrm{nV} / \sqrt{\mathrm{H}} \mathrm{z}$, resistance noise $0.4 \mathrm{nV} / \sqrt{\mathrm{H}} \mathrm{z}$ ).

To control the stability of the square voltage bias, a bridge was made of two resistors $(200 \Omega)$ and supplied at its two ends with two opposite square waves $(V=25 \mathrm{mV}$ peak-peak) delivered by two BEBOs. The signal at the middle point of the bridge was measured by a third BEBO, and its amplitude spectral density is displayed in Fig. 7. From 0.3 to $10 \mathrm{~Hz}$, the noise level of $1.5 \mathrm{nV} / \sqrt{\mathrm{H}} \mathrm{z}$ is as expected from the Johnson noise of the resistors in parallel and the pre-amplifier noise. Below $0.3 \mathrm{~Hz}$, this level slowly increases to reach $4 \mathrm{nV} / \sqrt{\mathrm{H}} \mathrm{z}$ at $0.01 \mathrm{~Hz}$. This level is still less than 5 to $50 \mathrm{nV} / \sqrt{\mathrm{H}} \mathrm{z}$, the range in which the intrinsic bolometer noise is expected to remain. Even if the noise of the bias sources is not negligible at very low frequencies, it will not be the main source of noise in the system.

To measure the total noise of the electronic system, a bolometer has been simulated by a resistor $(1 \mathrm{k} \Omega)$, a capacitance $(47 \mathrm{nF})$, and a low noise JFET $(1.5 \mathrm{nV} / \sqrt{\mathrm{H}} \mathrm{z})$. The values of the capacitance and the resistor have been calculated in order to produce the same Johnson noise at $T=300 \mathrm{~K}$ as a bolometer thermistor of $3 \mathrm{M} \Omega$ at $T=0.1 \mathrm{~K}$ (i.e. $4 \mathrm{nV} / \sqrt{\mathrm{Hz}}$ ). It can be seen on the noise spectrum (Fig. 8), that the electronic noise is approximately white, below $4 \mathrm{nV} / \sqrt{\mathrm{H}} \mathrm{z}$ above $0.2 \mathrm{~Hz}$, with a slight increase at lower frequencies reaching $15 \mathrm{nV} / \sqrt{\mathrm{H}} \mathrm{z}$ at $0.01 \mathrm{~Hz}$.

From the noise spectrum (see Fig. 9) obtained on a bolometer $\left(R_{\mathrm{B}}=40 \mathrm{M} \Omega\right)$ of the Diabolo experiment, the noise is approximately equal to $20 \mathrm{nV} / \sqrt{\mathrm{H}} \mathrm{z}$ (a $40 \mathrm{M} \Omega$ resistance has a Johnson noise about $15 \mathrm{nV} / \sqrt{\mathrm{Hz}}$ ) and stable down to $0.1 \mathrm{~Hz}$. The increase of the noise level below $0.1 \mathrm{~Hz}$ has been attributed to temperature fluctuations of the dilution stage because this noise appears to be very well correlated between the different bolometers and the thermometers. Subtraction of this noise using blind bolometers is possible and reduces the level by a factor of order 10.

Finally, the cross-talk between two channels of the full detection chain (from bolometer to digital output signal) has been measured on a bright planet (Saturn) to be less than $0.1 \%$, which includes both optical and electrical possible effects.

Independently of the Diabolo experiment, the use of the Thomson Inmos T805 to implement a digital lock-in detection has been successfully tested. Up to twelve channels have been simultaneously measured. The optimum digitizing rate needed to correctly analyze the signal has been tested. A rate corresponding to about 64 data points per bias period is enough to eliminate the transients. In the Diabolo system, the length of the residual transient is about 4 data points per half period, which have to be eliminated in the lock-in integration. The efficiency of this AC readout is therefore close to $88 \%$. Finally, the time needed to control the parameters of the bolometer readout by the 4 DACs was found to be equal to about $100 \mathrm{~ms}$.

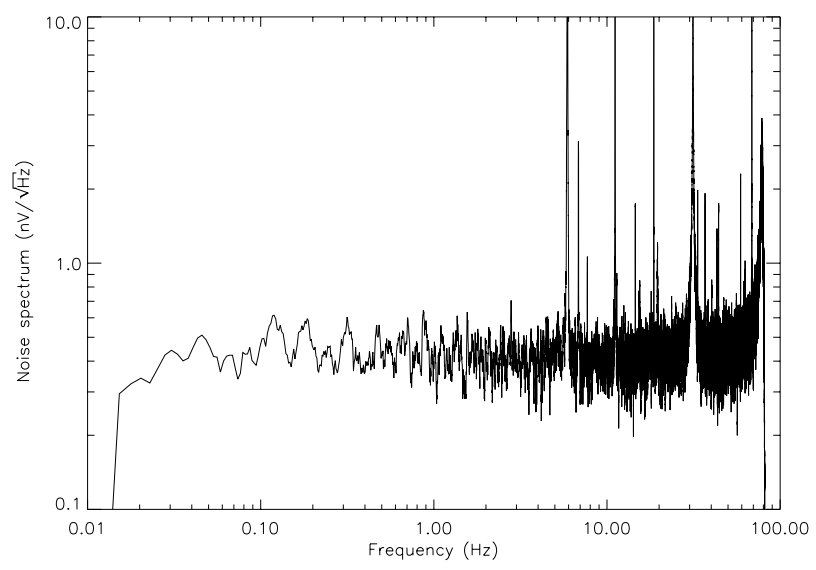

Fig. 6. Noise spectrum of the amplifier after lock-in $\left(f_{\text {modulation }}=81.38 \mathrm{~Hz}\right)$

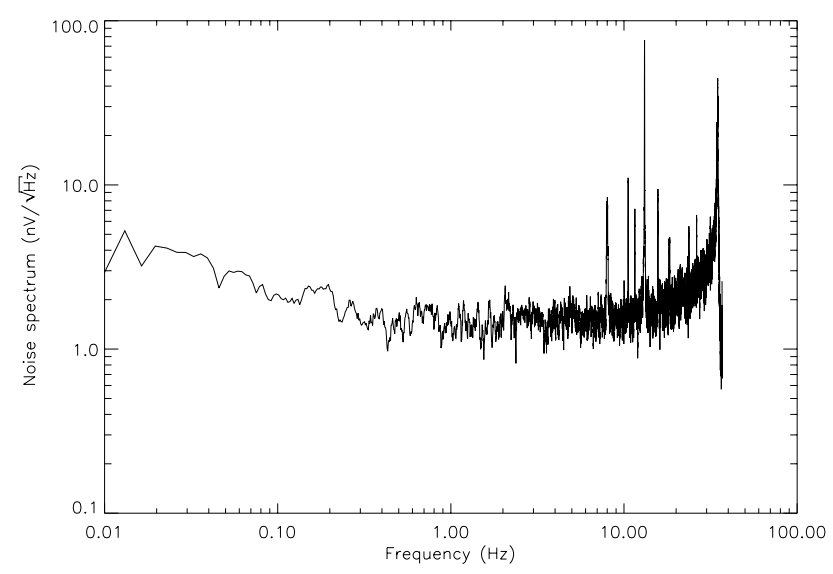

Fig. 7. Noise spectrum of the bias amplitude $\left(f_{\text {modulation }}=36.85 \mathrm{~Hz}\right)$

This system, tested and used on the Diabolo groundbased experiment at the focus of the IRAM 30 meter radio telescope in December 1995, allowed to observe the Sunayev-Zeldovitch effect at high angular resolution (a 30 arcsec beam and 3 arcmin beam throw) towards several clusters of galaxies (Désert et al. 1996).

\section{System for the COBRAS/SAMBA High Frequency Instrument}

The electronic architecture for the COBRAS/SAMBA High Frequency Instrument (HFI: 150 to $850 \mathrm{GHz}$ ), depends on the dimensioning of the system i.e. the final sampling frequency, the bias frequency, the rate and the dynamic of the ADC, and the rate of the modulator DACs actuation. The parameters linked to the practical implementation of the detection system, such as the parasitic capacitances, the wiring lengths, and so on are assumed 


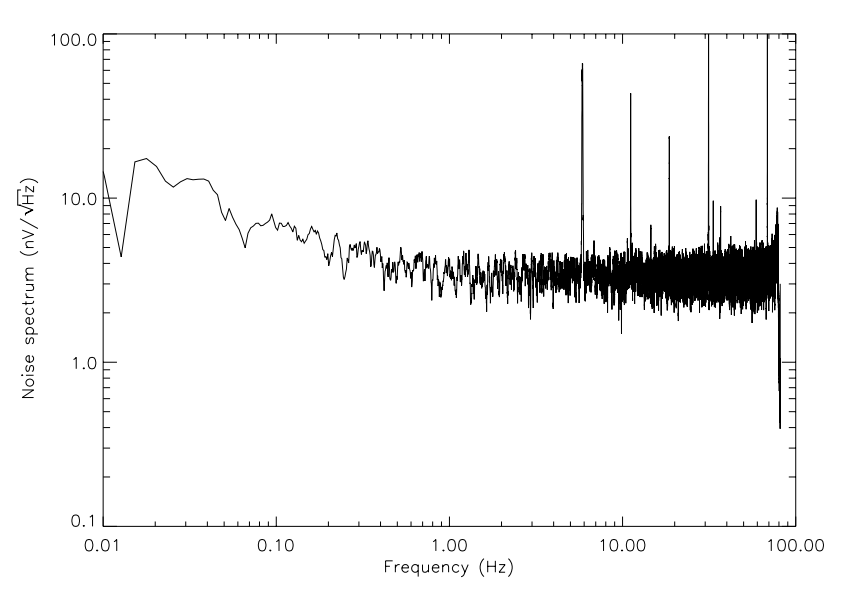

Fig. 8. Noise spectrum obtained with a bolometer simulator at $300 \mathrm{~K}\left(f_{\text {modulation }}=81.38 \mathrm{~Hz}\right)$

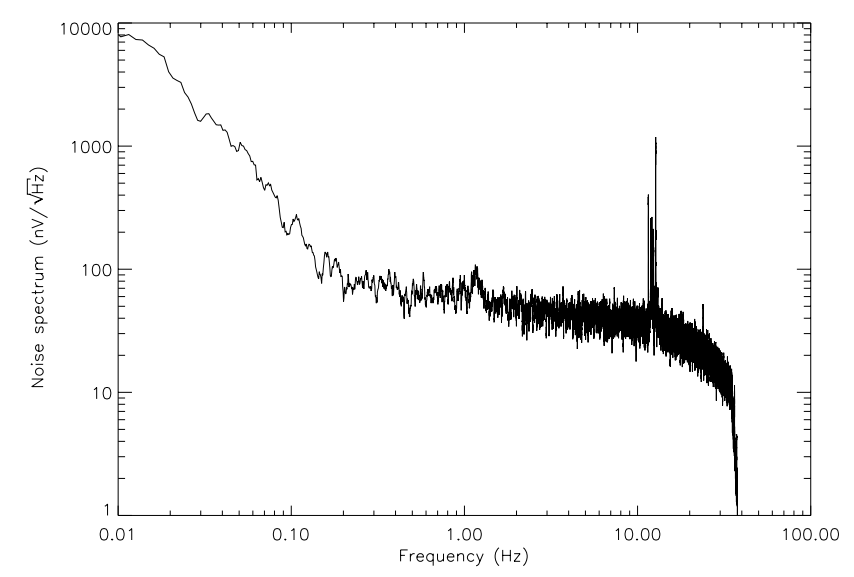

Fig. 9. Noise spectrum obtained with a bolometer $(R=40 \mathrm{M} \Omega)$ of the Diabolo experiment $\left(f_{\text {modulation }}=38 \mathrm{~Hz}\right)$

to be similar to those of Diabolo, since both instruments are based on the same cryostat that cools the bolometers down to $0.1 \mathrm{~K}$, i.e. an open loop dilution system (Benoît 1994).

From the technical specifications of COBRAS/SAMBA (see report on the phase A study 1996), the optimal choice of orbit is a Lissajous orbit around the L2 Lagrangian point of the Earth-Sun system. At this location, the Sun, the Earth, and the Moon are all located behind the payload, where their undesirable effects are at the lowest possible level in terms of flux. The scan angle, defined as the angle between the spin axis of the satellite and the telescope line-of-sight, is equal to $70^{\circ}$. So, the HFI beam will follow a circle of a $2 \pi \sin 70^{\circ}$ perimeter per minute of time, i.e. 340 arcmin per second.

The beam FWHM of the highest frequency channels is equal to 5 arcmin. To fulfill the Nyquist criterion and some safety margins (3 data points per beam), a sampling rate of 200 samples per second is needed. This is not the sampling rate of the ADC, but the one of the signal delivered to the on board computer, after lock-in detection. The electric bias frequency of the bolometer has to be an integer factor of this frequency. The digital lock-in allows the bias frequency to be equal to the scientific sampling rate.

To allow proper elimination of transients (see the end of Sect. 5), the rate of ADC is equal to 12.8 kilosamples per second (i.e. $64 \times$ bias frequency). The range of the ADC is determined by the ratio of the larger signal measured (a brightness temperature of about $100 \mathrm{mK}$ on bright sources) to the $\sigma / 2$ bolometer noise ( $\sigma=0.080 \mathrm{mK} \mathrm{rms}$ noise at $200 \mathrm{~Hz}$ sampling), i.e. 1216 , which is fulfilled with 12 bits.

The actuation rate of the DACs in the digital modulator can be chosen as lower than or equal to $0.0167 \mathrm{~Hz}$, synchronous with the satellite spinning rate. Indeed, due to the speed of the bolometer control by the 4 DACs (i.e. $100 \mathrm{~ms}$ ), the reset of the signal can, if necessary, be performed at each satellite rotation with a loss of only two samples. However tests on the Diabolo experiment show that using a temperature regulation of the $0.1 \mathrm{~K}$ stage allows, on clear sky conditions, to freeze the modulation parameters for several hours. The stability of a satellite system is expected to be still better since there is no atmospheric contamination of the background photon flux.

In order to meet these requirements, an architecture of the bolometer readout electronics of the COBRAS/SAMBA HFI (see Fig. 10) can be derived from the system developed and tested on the ground-based Diabolo instrument. As shown in Fig. 10, the elimination of transients, the signal lock-in, the filtering and the feedback control of the bias (through values delivered to the modulator DACs, controlling the shape and amplitude of the bias voltages) are performed by computers. Each computer is able to manage a group of about 12 bolometers, and delivers the processed signals to the on board computer (BEBA: Bolometer Electronic Box Assembly). There is one analog chain per bolometer (modulator with capacitive load, DACs, amplifier, ADC and digital command logic), and five computer units are needed for the 56 bolometers of the HFI. Bolometers belonging to the same wavelength channel will be dispatched to different computing units to avoid that a particular frequency become unavailable because of a computer failure.

This system is robust because it uses current space qualified technologies: the analog part is made of classical low noise electronics, the computer units are based on the Thomson Inmos T805 transputer. In addition to the processed signal, a fully sampled period of each bolometer is passed on to the telemetry at a slow rate. This will be used to check that the signal processing is correctly done, and to perform the first balance of the bias at the beginning of the mission. One of the advantages of the fully 


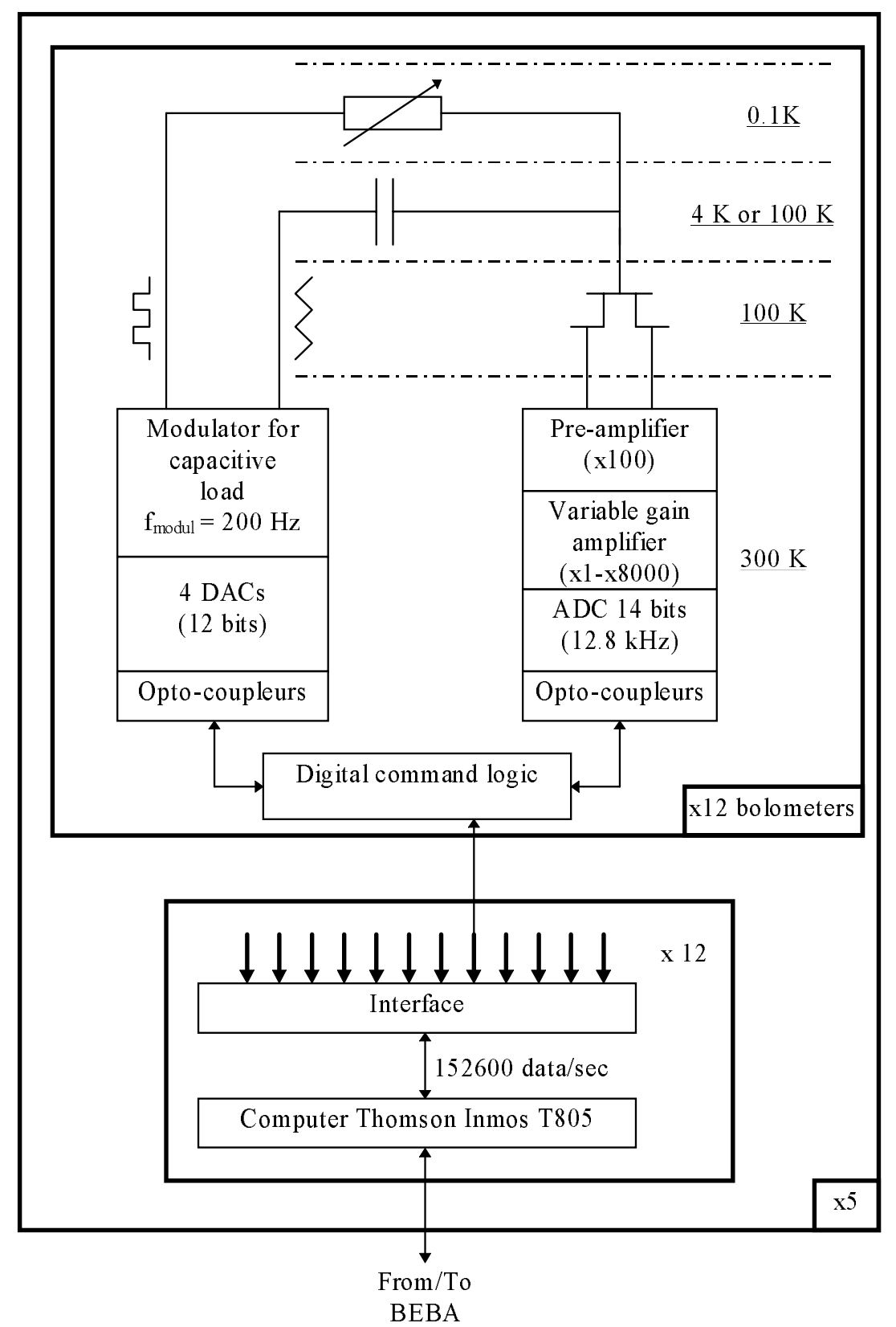

Fig. 10. Synoptic of the electronic system proposed for the COBRAS/SAMBA High Frequency Instrument

digital on board signal processing, with respect to a system using analog lock-in, is that it will be easier to handle in flight problems like an unexpected rate of particle induced spikes or an anomalous background power on the detectors.

In addition, in flight measurements of the bolometer $V(I)$ characteristic are possible. This will allow to find the optimum working point of each bolometer which will depend on the background photon flux. This parameter is difficult to predict before the mission, and it may induce high costs if it is specified within a narrow range (cleanness of the optics). Moreover, recording the bolometer current and voltage allows to deduce the absolute optical power on the bolometer, which gives a calibration of the bolometer response. 


\section{Conclusions}

A new bolometer readout system has been developed and tested. It consists of an analog low signal proximity circuit and an amplifier, a "modulator" generating two bias signals, a digital command logic, and a digital to analog converter transmitting raw data to the control computer. Original features at all stages of this system give it unique performances. 1) The periodic nature of the bolometer bias translates the measurement frequency outside of the low frequency noises of electrical origin. In consequence, measurements of radiations varying at very low frequencies (down to $0.1 \mathrm{~Hz}$ or less) are possible without degradation of the sensitivity. 2) Two balanced bias signals are put at the two ends of the bolometer-load bridge, which allow to adjust around zero the mean voltage of the middle point. In this way, the dynamics needed for the amplification chain is considerably reduced compared to a system which will amplify the full voltage of the bolometer (Lange et al. 1996). We are able to measure the total power on a single pixel even if the background photon flux from the telescope is important (e.g. COBRAS/SAMBA). 3) The bias current in the thermistor is a square wave, keeping the electrical power deposited in the bolometer constant, and allowing to choose the bias frequency independently of the bolometer thermal characteristics. 4) The value of the bias signals is computer controlled, which gives the complete knowledge of the resistance of the bolometer thermistor. The measurement of the power background on the bolometer is then insured. 5) The load impedance in the measurement bridge is implemented with a capacitor. This choice eliminates the additional Johnson noise produced by the traditional resistive load. It also allows to locate the load at intermediate temperatures, such as $4 \mathrm{~K}$ or $100 \mathrm{~K}$, which limits the number of wires per bolometer to 2 , instead of 3 with a conventional circuit. It reduces in the same proportion the heat load by the wiring conduction on the most critical cryogenic stage. 6) All the bias parameters are computer controlled, allowing to face unpredictable situations, and insuring an optimal use of bolometers all along the life of a space project.

This system has been experimentally tested and used on the Diabolo ground-based astronomical experiment. The results of these tests allowed to validate the electronic system with the Diabolo bolometers, i.e. to demonstrate the very good stability of the bias and the low noise of the system.

The electronic architecture for the bolometer bias and readout on the COBRAS/SAMBA HFI, described in
Sect. 6, uses the specificity of the electronic system developed on the ground-based Diabolo instrument and modified for space borne application, i.e. the digital modulator with capacitance load and the digital lock-in fully controlled by a space qualified computer based on the Thomson Inmos T805 transputer. This system has also been experimentally tested on the Diabolo experiment, and demonstrated its ability to work under operational conditions. The performances of this new readout system open new modes of measurements to instruments using bolometers, such as the slow scanning of the sky with independent pixels, as featured in COBRAS/SAMBA.

Acknowledgements. We would like to thank the referee, H. Murakami, for his fruitful comments. The support by CNES of S. Gaertner and M. Piat is gratefully acknowledged. The Diabolo experiment has been funded by INSU and the technical research associated with its development has been supported by CNES and by the different laboratories of the authors. We thank the group of Spectrométrie Thermique of IAS for the study and realisation of the bolometers used in this study, under the scientific responsibility of N. Coron. Finally, S. Gaertner gratefully aknowledges support from the TTI department of Alcatel Espace, and more precisely P. Oudard and C. Lesthievent.

\section{References}

Benoît A., Bradshow T., Orlowska A., Jewell C., Maciaszek T., Pujol S., 1994, SAE Technical Paper Series 941276

Benoit A., et al., 1996 (in preparation)

Bersanelli M., et al., 1996, ESA D/SCI(96)3

Coron N., 1976, Infrared Phys. 16, 411-419

Désert F.-X., et al., 1996 (in preparation)

Devlin M., Lange A.E., Wilbanks T., Sato S., 1993, IEEE Trans. Nuclear Sci. 40, 162-165

Jones R.C., 1953, J. Opt. Soc. Am. 43, 1-14

Low F.J., 1961, J. Opt. Soc. Am. 51, 1300-1304

Lange A.E., Church S.E., Mauskopf P.D., Bock J.J., DelCastillo H.M., Beeman J., Ade P.A.R., Griffin M.J., 1996, in: Proc. 30th ESLAB Symp., Rolfe E.J. (ed.), p. $105-110$

Low F.J., Hoffman A.R., 1963, Appl. Opt. 2, 649-650

Mather J.C., 1982, Appl. Opt. 21, 1125-1129

Mather J.C., 1984, Appl. Opt. 23, 584-588

Murakami H., et al., 1996 (to be published in PASJ)

Rieke F.M., Lange A.E., Beeman J.W., Haller E.E., 1989, IEEE Trans. Nuclear Sci. 36, 946-949

Wilbanks T., Devlin M., Lange A.E., Sato S., Beeman J.W., Haller E.E., 1990, IEEE Trans. Nuclear Sci. 37, 566-572 